\title{
TV/Series
}

15 | 2019

La Sérialité en question(s)

\section{La sérialité aux bords de la fiction télévisée, ou l'intermédialité comme mise en crise du « tout narratif » sériel}

\section{Saute-Requin}

\section{(2) OpenEdition}

Journals

Édition électronique

URL : http://journals.openedition.org/tvseries/3724

DOI : 10.4000/tvseries.3724

ISSN : 2266-0909

Éditeur

GRIC - Groupe de recherche Identités et Cultures

Référence électronique

Saute-Requin, "La sérialité aux bords de la fiction télévisée, ou l'intermédialité comme mise en crise du « tout narratif » sériel », TV/Series [En ligne], 15 | 2019, mis en ligne le 16 juillet 2019, consulté le 20 juillet 2019. URL : http://journals.openedition.org/tvseries/3724 ; DOI : 10.4000/tvseries.3724

Ce document a été généré automatiquement le 20 juillet 2019.

\section{(c) $(1) \odot$}

TV/Series est mis à disposition selon les termes de la licence Creative Commons Attribution - Pas d'Utilisation Commerciale - Pas de Modification 4.0 International. 


\title{
La sérialité aux bords de la fiction télévisée, ou l'intermédialité comme mise en crise du «tout narratif » sériel
}

\author{
Saute-Requin
}

1 L'étude des séries télévisées se porte bien, comme l'attestent les perspectives nombreuses et diverses qui permettent aujourd'hui de les aborder: études sociologiques, économiques, narratologiques, génétiques, gender, philosophiques, etc. Pourtant, à y regarder d'un peu plus près, il semble que ces recherches souvent stimulantes reposent fréquemment sur la mise en valeur du fonctionnement des fictions qu'elles dépeignent, des personnages qui les habitent, de leurs thématiques, de leur structure, ou encore de leurs procédés d'écriture. Si la question de la mise en scène est parfois invoquée, conférant à certaines productions une identité audiovisuelle forte, cette approche plus frontalement esthétique demeure profondément marquée par l'héritage des codes de représentation et d'analyse forgés dans le champ du cinéma. En d'autres termes, si les séries télévisées sont parfois - mais pas toujours - analysées comme des formes esthétiques, ce sont principalement des outils issus d'une autre discipline et d'un autre champ médiatique qui permettent d'en faire l'analyse.

Ce texte, issu d'un travail collectif mené par un groupe d'enseignants-chercheurs et d'étudiants de l'université Rennes 2 venus de plusieurs disciplines (Lettres, Cinéma, Sciences de l'information et de la communication), a vu le jour à l'aune d'un constat: celui d'une prévalence, dans la réflexion universitaire sur les séries télévisées, de modèles principalement narratologiques ou, à tout le moins, d'une prise en considération de ces objets qui met au premier plan leur dimension narrative, au détriment d'autres prismes d'étude possibles.

3 En effet, au-delà des composantes audio-visuelles des séries télévisées, qu'en est-il de la sérialité en elle-même, des inventions qu'elle autorise mais aussi des conceptions dont elle procède ? Pourquoi la sérialité télévisuelle ne pourrait-elle pas faire l'objet d'analyses 
(de type plastique, structurel, rythmique, etc.) qui ne passeraient ni par le prisme de la "mise en scène", ni par celui de la narration? La question mérite d'autant plus d'être posée que de nombreuses œuvres appartenant à d'autres champs artistiques (photographie, peinture, musique, poésie, danse, sculpture, etc.) ont pu être interrogées au prisme de leur caractère sériel, sans pour autant proposer ou présupposer quelque récit que ce soit. En s'intéressant à la notion même de sérialité, d'un point de vue intermédiatique, et aux discours qui la promeuvent à des époques et dans des contextes divers, un tel constat s'avère d'autant plus frappant, et incite à se demander pour quelles raisons la télévision semble ainsi faire écran à d'autres conceptions de la sérialité, qui ne reposeraient pas exclusivement sur ce que l'on pourrait appeler un paradigme narratocentré.

En ce sens, cet article se donne un triple objectif : tout d'abord essayer de comprendre les raisons pour lesquelles la sérialité télévisuelle se trouve aussi fortement rattachée au modèle narratif. Puis, ouvrir la perspective en nous interrogeant sur les origines du terme de "série» dans le champ médiatique, pour montrer que si son appréhension contemporaine est largement conditionnée par le modèle des séries télévisées, cette perspective est loin d'être la seule possible, et que la notion ouvre sur des horizons esthétiques parfois bien éloignés de la narrativité. Enfin, à partir de ce regard tourné vers le passé, nous lancerons quelques pistes visant à proposer d'autres approches possibles de la sérialité télévisuelle, en nous demandant ce que pourrait produire une analyse nourrie par des cadres interprétatifs empruntés à d'autres médias sériels.

Envisager frontalement ces questions, c'est ouvrir une boite de Pandore. Aussi faut-il prendre ce texte pour ce qu'il est: un chantier de travail, un faisceau d'interrogations et de propositions, qui ne prétend pas être exhaustif, ni définitif, et encore moins délivrer une "vérité " demeurée jusqu'ici invisible. Notre ambition est, plus modestement, d'attirer l'attention sur quelques points de méthodologie de la recherche, et de suggérer quelques pistes de réflexion, qui nous semblent injustement mésestimés, mais potentiellement féconds. D'où la forme particulière que nous avons voulu donner à ce texte : écrit à quatorze mains ${ }^{1}$, ouvrant sur des perspectives parfois bien différentes, il est à prendre comme un état des lieux, sans doute lacunaire, et comme un point de départ à la construction collective d'un questionnement que semble susciter, de plus en plus, l'intermédialité culturelle dans laquelle s'inscrivent les séries contemporaines.

\section{Pourquoi la narration?}

Si les séries télévisées des trente ou quarante dernières années ont appris à soigner leur esthétique audiovisuelle, elles demeurent néanmoins appréhendées avant tout à partir de leur contenu narratif, c'est-à-dire des histoires qu'elles racontent. Car si l'ambition formelle des séries a pu contribuer à attirer un nouveau public, c'est bien par le caractère souvent vertigineux de leurs narrations, par une complexité liée à la forme sérielle même, qu'elles ont su se démarquer du cinéma et s'attirer le succès public et critique qu'elles connaissent aujourd'hui. Dans les années 1990 émerge la notion de "Quality TV" ", approfondie et théorisée par Jason Mittell, qui parlera quant à lui de «Complex TV ». Or, cette complexité est d'abord narrative. Elle consiste notamment à brouiller et à mixer les logiques feuilletonesques et sérialisantes, ce dont une sitcom comme Friends (NBC, 1994-2004), où alternent sketches comiques apparemment gratuits et feuilletons amoureux au long cours, peut attester. L'architecture du récit s'en trouve raffinée, 
s'approchant de plus en plus d'une temporalité humaine. Cette temporalité complexe du récit, basée sur l'agencement d'arcs narratifs qui dessinent un univers fictionnel riche, allait nécessairement intéresser la narratologie, et plus largement les théories de la fiction.

7 Le récit télé-sériel, qui émerge au début des années 1950, est issu de «l'apparition de nouveaux arts narratifs rendus possibles par les progrès techniques ${ }^{4} "$, notamment le cinéma et la bande dessinée, qui avaient émergés à la fin du $\mathrm{XIX}^{\mathrm{e}}$ siècle. Si Thierry Groensteen les qualifie d'emblée de "narratifs ${ }^{5}$ », c'est qu'ils se sont peu ou prou concentrés sur le principe de narration fictionnelle, fonction de l'art que remettent en question, à la même époque et sous l'impulsion des avant-gardes, les arts traditionnels. À l'instar de leurs prédécesseurs, les séries télévisées naissent (et se développent) du croisement et de la construction réciproque des genres fictionnels de la culture populaire et de l'industrialisation des productions culturelles, processus qui débute avec l'explosion de la presse au milieu du XIX ${ }^{e}$ siècle, que la télévision renforce, et qui a encore cours aujourd'hui dans bien des pratiques culturelles (jeux vidéo, jeux de rôles, fanfiction, etc.).

Or, si, pour ces modes d'expression, « la narration s'est très rapidement imposée comme leur pente naturelle, leur vocation objective ${ }^{6} "$, la sérialité s'est imposée comme la meilleure façon de dévaler cette pente et d'accomplir cette vocation, c'est-à-dire, dans ce contexte de généralisation du modèle capitaliste, de satisfaire une demande. Le phénomène est sans doute imputable au contexte d'industrialisation dans lequel il voyait jour: la production en série, nouvelle panacée pour satisfaire aux besoins humains, à partir du moment où elle s'est appliquée aussi aux objets se prêtant à une consommation culturelle, devait finir par se répercuter sur le contenu même de ces objets. Le caractère sériel de la production matérielle de l'objet (roman-feuilleton, film de cinéma, livre, et sans doute peut-on étendre cette liste à bien des objets de consommation culturelle moins directement liés à la fiction) touche la fiction dont il est porteur, dans sa forme et son contenu : la narration proprement dite autant que l'univers fictionnel qu'elle déploie sont transformés par le paradigme de la sérialité, lequel modifie à terme la relation du consommateur à ces objets culturels, à leur support particulier, à leurs modes d'adresse, ainsi qu'à la fiction, qui transcende ces derniers, voire, parfois, les occulte ${ }^{7}$. Parce qu'elles sont au cœur de ces problématiques idéologiques, économiques, anthropologiques et épistémologiques, les séries télévisées nous apparaissent comme le parangon de ce paysage culturel qui associe narration fictionnelle et sérialité à des fins commerciales. La fiction se sérialisant, c'est-à-dire, faisant subir à ses narrations une mise en ordre sérielle, et ce dans la plupart de ces arts issus de la logique industrielle, on comprend mieux dès lors que les conceptions aujourd'hui les plus répandues de la sérialité, notamment à la télévision, aient embrassé en premier lieu des approches narratologiques, auxquelles se sont adossées des théories de la fiction.

9 Ces approches ont par ailleurs été nécessaires dans le processus de légitimation des séries télévisées. Une fois dépassée l'approche de la télévision comme phénomène essentiellement social (son rapport aux discours dominants, ses effets prétendus sur le téléspectateur), il a bien fallu constater, dans un grand nombre de ces « fictions plurielles ${ }^{8}$ ", la qualité esthétique de ces productions issues des industries culturelles tant décriées par les milieux intellectuels, de Sainte-Beuve à l'École de Francfort ${ }^{9}$. Or, cette reconnaissance n'est pas venue des chercheurs en études audiovisuelles ${ }^{10}$, mais des américanistes, des sociologues de la culture et des chercheurs en information et communication, qui étudiaient déjà les productions médiatiques et qui ont déployé un 
éventail d'outils issus de disciplines variées. Ainsi l'approche narratologique de ces objets audiovisuels a pu être privilégiée précisément parce que ceux qui s'y sont intéressés n'étaient pas spécialistes d'études audiovisuelles ou parce qu'ils adoptaient auparavant une perspective narratologique sur le cinéma ${ }^{11}$. Cette approche peut s'enrichir de questionnements liés à la fiction, ou de questionnements davantage sociologiques, hérités des études culturelles ${ }^{12}$. Cette narratologie élargie, combinant une approche thématique et modale, analyse la fiction comme communication construite, et relève d'une compétence sémiotique largement partagée dans le domaine des sciences humaines, ce qui explique qu'un certain nombre de disciplines commencent à étudier les séries télévisées (leur contenu narratif tout du moins), comme objets audiovisuels certes, mais surtout fictionnels, fondés sur des représentations.

De fait, si la réception et l'analyse des séries télévisées restent très largement subordonnées à leur dimension narrative, c'est qu'elles ne semblent pas faire autre chose que raconter des histoires, art qu'elles célèbrent fréquemment, par exemple en représentant des scènes de "bedtime story» (Doctor Who, Master of None, Shameless (UK), The Office (US), Community ${ }^{13}$, etc.). Cette idée est également rappelée par l'avertissement liminaire de la série Fargo (FX, 2014-) : "This is a (true) story ». C'est pourquoi, dans cette perspective, les séries télévisées, au-delà d'un spectacle audiovisuel, peuvent être envisagées comme un art de la relation (fictionnell ${ }^{14}$ ) : l'objectif principal étant de captiver le téléspectateur, on lui propose un objet audiovisuel qui lui raconte une histoire et, ainsi, crée un lien avec lui. Ce lien s'établit dans la durée et avec une certaine régularité, renforçant par là même l'idée de relation. Il s'établit à travers l'histoire racontée, le monde fictionnel et son devenir, mais aussi par la façon spécifiquement audiovisuelle de raconter cette histoire. Ainsi, écrit Jean-Pierre Esquenazi, «tout récit tisse, au fur et à mesure de sa progression, l'image d'un monde qui l'abrite ${ }^{15}$. " Quelle est cette image, et comment le récit la tisse-t-elle? Cette double question, appelant l'utilisation combinée d'une narratologie thématique et modale, doit inciter l'analyste à considérer tout autant le contenu et l'ordonnancement du récit que sa mise en scène audiovisuelle, celle-ci participant, en premier lieu, à construire la relation du spectateur à la fiction.

Il y a donc un double enjeu qui s'attache selon nous, dans un tel cadre, aux séries télévisées. En premier lieu, afin d'appréhender la complexité narrative propres aux créations sérielles contemporaines issues du média télévisuel, il est nécessaire de «tenir compte des nouveaux paradigmes développés dans le champ de la théorie du récit ${ }^{16}$.» Néanmoins, si la narratologie porte à ce point son attention sur ce type d'objet, c'est aussi parce qu'il permet d'envisager à nouveaux frais le récit lui-même, à le «repenser » en retour ${ }^{17}$ afin de saisir de nouvelles spécificités narratives.

Le second enjeu concerne plus spécifiquement les théories de la fiction. En effet, au sein de ce qu'on qualifie parfois d'ère de la fiction généralisée, dans laquelle nous serions aujourd'hui plongés, « liant l'appréhension collective des objets fictionnels au territoire du ludique et à l'infinité des possibles ${ }^{18}$ ", nous pensons que les séries télévisées " évolutives »- autrement dit feuilletonnesques - occupent une place déterminante. Elles permettent de configurer de véritables «mondes possibles » du fait de l'extension assez considérable de leur continuum. C'est ainsi l'œuvre même qui, en tant que telle, devient un monde dans lequel « les créateurs [...] vont jusqu'à investir dans leurs intrigues des reflets directs des questionnements ontologiques et des possibles réflexifs soulevés par les théoriciens, exploitant sous tous les angles la métaphore du texte-univers ${ }^{19}$ ». Si l'on considère ici les nouvelles pratiques transmédiatiques qui émergent de manière 
croissante depuis deux décennies, selon lesquelles un univers fictionnel est déployé puis développé sur différents supports - produisant, comme le note Matthieu Letourneux, un « effet de série ${ }^{20} »-$, il semble que la propension de ces univers diégétiques à être déclinés transmédiatiquement ${ }^{21}$ soit accrue " quand les objets étaient déjà sériels auparavant ${ }^{22}$ ». Des expériences transmédiatiques comme celles de Lost (ABC, 2004-2010), en ce qu'elles visent entre autres à la complétion et à la suturation des béances fictionnelles inhérentes au caractère fragmentaire de son récit, se structurent ainsi intégralement autour de la série télévisée originelle.

13 Toutefois, et quand bien même le prisme de la narrativité serait le plus "naturel » ou le plus logique pour aborder ces objets télévisuels, cette synergie entre narrativité et sérialité n'a-t-elle pas progressivement contribué à occulter certaines dimensions fondamentales de la création sérielle? N'a-t-elle pas fait oublier ce qu'est aussi, et par ailleurs, une création en série?

\section{Pour une archéologie de la série}

14 En effet, la sérialité n'est pas un phénomène (ou un processus) exclusivement narratif. Le retour à l'étymologie peut s'avérer éclairant à telle enseigne, comme peut en témoigner le travail de Matthieu Letourneux. Selon lui, le latin series « renvoyant à la fois à l'idée d'une suite ininterrompue, d'un arrangement de choses qui se tiennent et d'entrelacs, de fils ou de tracés - manifeste l'empan du terme, entre ensemble fini et indéfini, modèle de la trame et de la ligne, juxtaposition et organisation ${ }^{23} »$. C'est sur le verbe serere (tresser) que se forme le substantif, appuyant les métaphores du tressage ${ }^{24}$ et du tissage ${ }^{25}$ dans les approches narratologiques. Dans ses usages concrets, le latin series ne renvoie pas tant à l'idée de succession qu'à une mise en réseau spatiale d'éléments plus ou moins discontinus. Quinte-Curce l'emploie au sens d'« enchevêtrement ${ }^{26} »$ dans la description d'une chaîne de montagnes: la proximité et les intersections des chaînes en font un ensemble avec sa cohérence ( cohcrentia ») propre. Il en est de même pour la description d'un enchevêtrement de nœuds sur le joug du char de Gordius ${ }^{27}$, donnant lieu à l'expression "nœud gordien ». Ailleurs, series s'entend comme un réseau, de vaisseaux sanguins chez Pline l'Ancien ${ }^{28}$, de routes chez Stace ${ }^{29 .}$

15 C'est davantage dans ses acceptions abstraites que series est recouvert par une logique narrative et qu'il désigne des ensembles marqués par la succession logique ou chronologique. Tout d'abord, dans l'ordre du discours rhétorique ou philosophique, il revêt le sens de mise en ordre ou de structure argumentative. Ainsi Quintilien regrette-til que les nouveaux orateurs utilisent des structures rhétoriques prototypiques, qui entravent leurs discours dans des argumentations qui forment une « série inextricable ${ }^{30}$ ». Dans ses acceptions abstraites, la sérialité s'appuie logiquement sur la cognition et un enchaînement causal. Ce n'est finalement que lorsque cet enchaînement causal concerne une temporalité, une mise en récit chrono-logique, qu'il revêt une dimension narrative : le terme apparaît lorsque Cicéron définit le destin (fatum) comme un enchaînement causal d'événements, où series signifie succession ordonnée, chronologique ${ }^{31}$. Autrement dit, la sérialité est narrative lorsqu'elle concerne une suite d'événements perçus comme causés entre eux et lorsque la jonction narrative entre ces événements prend le pas sur des modes d'interconnexion plus concrets. 
16 De surcroît, d'autres termes comme «saison » ou «épisode ", qui peuvent désigner aujourd'hui des ensembles narratifs, ont acquis récemment cette dimension narrative à l'aune du développement des formes télévisuelles ${ }^{32}$ et d'un vocabulaire spécialisé, désormais entré dans le langage courant. Ce penchant vers la narrativité s'explique par d'autres facteurs, notamment la massification des pratiques culturelles et le développement de pratiques sérielles visant à fidéliser les consommateurs dans une logique capitalistique, où la fragmentation et les logiques transtextuelles prennent le pas sur l'unicité de l'œuvre. Cela donne lieu à des glissements sémantiques qui rendent compte de la tension entre unités de diffusion autonomes et continuité narrative compensatoire. Ainsi le mot «feuilleton » se forme sur le format de publication, et ce n'est que plus tard qu'il vient à désigner un mode de continuité narrative, qui contrebalance la fragmentation éditoriale. À l'inverse, " épisode », originellement intégré dans un récit unique comme «partie complète de la tragédie qui se situe entre les chants complets du chœur ${ }^{33}$ ", s'autonomise en devenant une unité éditoriale et narrative, tout en étant intégrée dans une série.

Pour ce qui est du terme "série ", il semblerait que sa compréhension essentiellement narrative, au détriment de la richesse d'autres acceptions possibles, soit due à deux types de contaminations. D'une part, l'entrée de «série " en français dans le vocabulaire mathématique (entre la fin du XVII et le début du XVIII ${ }^{\mathrm{e}}$ siècle) repose sur une acception quasi-identique à celle du terme "suite ${ }^{34}$ ", si bien que L'Encyclopédie de Diderot et D’Alembert les regroupe dans la même entrée ${ }^{35}$. Cela pourrait expliquer une confusion durable entre les deux mots et des échanges sémantiques, par lesquels les usages courants de "suite " deviendraient des acceptions de "série ». "Série " entre dans le champ lexical artistique tardivement au $\mathrm{XIX}^{\mathrm{e}}$ siècle en France. À cette époque les termes « roman-fleuve » et «feuilleton» dominent dans le champ littéraire pour qualifier les publications romanesques sérielles bénéficiant d'une continuité narrative ${ }^{36}$. Le second motif de cette contamination sémantique serait l'effet du terme anglais "serial », formé sur le substantif « series ». Il est utilisé dès 1841 comme adjectif (« serial publication ») et en 1846 comme substantif pour qualifier des feuilletons littéraires, alors que la même année «serialist» est attesté pour qualifier les feuilletonistes ${ }^{37}$. Même si la formation du mot anglais repose sur le mode de publication, le glissement sémantique de "série » vers la narrativité dans la sphère artistique s'expliquerait en partie parce qu'il renvoie au «feuilleton» en français. Cette narrativisation du terme occulte le fait que le développement de la sérialité dans la sphère artistique correspond avant tout à une évolution des pratiques liée à l'industrialisation du champ artistique et culturel.

À ce titre, on ne peut que constater la systématisation des usages du terme "série » dans le courant $\mathrm{du} \mathrm{XIX}^{\mathrm{e}}$ siècle, dans une acception évidemment différente de celle que tend à façonner notre imaginaire contemporain. Le terme désigne bien, alors, un mode de production et - par extension - un mode de consommation des formes artistiques, reposant moins sur l'unicité de l'œuvre que sur sa reproductibilité. Dans un sens encore distinct de celui que l'on peut trouver au sein des usages mathématiques, ou dans le cadre de la musique (reposant sur la construction d'une logique successive, la mémoire des notes jouées conditionnant l'effectuation des notes suivantes en vue d'élaborer une harmonie rythmique et mélodique ${ }^{38}$ ), la notion de série désigne rapidement la reproduction mécanique d'œuvres, permettant leur diffusion à grande échelle, suivant ce qui se pratiquait déjà dans la production de monnaies dès l'Antiquité, ou le développement de l'imprimerie $\mathrm{au} \mathrm{XV}^{\mathrm{e}}$ siècle. D'où son rattachement assez évident à la 
presse et à la photographie notamment, mais également à la diapositive. Or, si la série ne désigne dans ce contexte qu'un mode de production ${ }^{39}$, ce dernier a clairement un impact sur l'imaginaire artistique de l'époque. De fait, au-delà de la reproductibilité des œuvres, on trouve l'idée d'une forme de logique dans la reproduction, reposant soit sur "l'identicité » des objets produits, soit sur leur proximité, convoquant le principe de variation sur un thème par exemple, et déterminant des pratiques de collection. Là encore, les lieux concernés sont nombreux, allant de la philatélie ${ }^{40}$ aux vues photographiques en passant par la peinture avec les séries de toiles comme celles de Claude Monet par exemple, variations sur des motifs choisis peints à différentes heures de la journée (Nymphéas, Cathédrales, etc. ${ }^{41}$ ), ou également les séries de portraits, conception picturale qui n'apparait certes pas avec la modernité, mais qui ne se verra jamais concrètement nommée comme telle avant le $\mathrm{XIX}^{\mathrm{e}}$ siècle. Par ailleurs, la diffusion à grande échelle de certains objets (ouvrages, reproductions d'images, presse, etc.) ouvre sur une dimension économique s'inscrivant dans la continuité d'un capitalisme dit " moderne ", lié à la révolution industrielle, incitant à fidéliser ce public pour assurer des bénéfices sur le long terme.

De fait, c'est suivant cette même logique que l'on va observer, à la fin du XIX siècle et au début $\mathrm{du} \mathrm{xx}^{\mathrm{e}}$, une conception similaire du terme dans le domaine des vues animées, notamment les vues cinématographiques. Au-delà du principe de «mise en série » de photographies qui constitue le fonctionnement même de ces objets, voire des premiers programmes cohérents constitués de séries de vues ${ }^{42}$, remarquons que cette idée de cohérence se voit rapidement suivie d'un autre principe, lié à la fidélisation du public via des acteurs/personnages récurrents. Pour ne prendre qu'un exemple, la revue CinéJournal explique dès 1908 que « la société française des Films Éclair, attentive à suivre le goût du public, a commencé la série des aventures extraordinaires de Nick Carter ${ }^{43}$ ". L'article va même plus loin, estimant que « le succès que remporte la première partie [...] montre bien que la cinématographie [...] peut connaître la même faveur que le roman, le feuilleton et le théâtre ${ }^{44} »$. Une telle affirmation ne fait que confirmer l'impact d'un imaginaire lié à ces formes artistiques sur la vision contemporaine de la sérialité.

Ces premiers exemples d'une volonté de fidélisation des spectateurs se répercutent par la suite dans un grand nombre de serials, des Fantômas de Louis Feuillade en France (1913-1914) aux Exploits of Elaine de Louis Gasnier aux États-Unis (1914-1915). Il est intéressant de constater que c'est à l'aune de ces productions particulières que tout un vocabulaire lié à la sérialité narrative apparait, comme peut par exemple en témoigner la réception américaine des Vampires de Louis Feuillade : le Motion Picture News évoque ainsi le «troisième épisode » de ce qu'il nomme un " photo-novel ${ }^{45}$ » (encore l'influence du roman), mais également des "twists of plots » (retournements de situation), ou encore une "succession de surprises rapides" dans ce qui prend bien le nom à l'époque de " $\operatorname{série}^{46}$ ». Mais on le voit, ce regard sur la sérialité est bien hérité d'une approche singulière liée à des formes avant tout narratives (feuilleton, théâtre, roman), que l'on lie à l'idée de successivité - mais il n'est en aucun cas lié à la notion de série elle-même qui, nous l'avons compris, a pu tout à fait désigner d'autres formes de production, non limitées à la narration (peinture, photographie, musique, etc.), voire, qui ambitionnaient même à l'époque de détruire la narration. Il conviendrait dès lors d'explorer plus avant les tours et détours de cette contamination des formes sérielles par l'imaginaire narratif telle qu'elle se dessine dans le courant du $\mathrm{xx}^{\mathrm{e}}$ siècle, au-delà même de la seule télévision ; 
mais aussi d'envisager les formes de persistance ou de survivance de la sérialité en dehors du paradigme narratif.

\section{Sérialité ouverte}

21 La fortune publique et critique des séries télévisées n'a donc pas seulement consacré un modèle dominant - celui de la narrativité -, et presque hégémonique, au détriment d'autres modèles possibles : elle a aussi installé une conception générale de la sérialité qui, prenant le pas sur les autres, oblitère l'historicité de la notion, et ce faisant, empêche peut-être le renouvellement de ses usages. En ce sens, on peut dire que la consécration des séries télévisées a enclenché un mouvement tautologique, qui est selon toute vraisemblance le signe avant-coureur d'une entropie et d'un épuisement à venir. Ainsi, dire que la série reprend le flambeau du cinéma (et avant lui, du roman) comme grand art du récit, c'est donc accepter d'emblée l'idée - contestable - que le cinéma est d'abord et toujours un art du récit.

Ouvrir l'analyse des séries télévisées à d'autres approches impose donc de se départir, au moins partiellement ou provisoirement, de la logique narrato-centrée. Un tel programme, dont on n'esquissera ici que les très grandes lignes, pourrait consister tout d'abord à convoquer des conceptions de la sérialité antérieures ou concurrentes au modèle narratif, ce qui revient au fond à contester plus largement l'idée selon laquelle la narrativité est inhérente à la condition des images en mouvement. Il s'agirait ainsi de proposer, d'importer ou de réactiver des modèles d'analyse dans lesquels la narration peut certes tenir un rôle, mais pas plus important, et surtout, pas plus structurant que d'autres dimensions qui traversent aussi les objets télévisuels, et sont paradoxalement négligées aujourd'hui.

Rappelons tout d'abord qu'il existe en cinéma, même si elles sont loin d'y être majoritaires, des formes absolument non-narratives, et même des formes sérielles nonnarratives, c'est-à-dire des séries de films, explicitement conçues comme telles, et dans lesquelles la narration n'est ni un prérequis pour les cinéastes, ni un horizon pour les analystes. On les rencontre essentiellement dans le champ de ce qu'on a coutume d'appeler le cinéma " expérimental ». Ce sont, parmi bien d'autres, la série des Bouquets de Rose Lowder (1994-2009), les Persian Series (1999) de Stan Brakhage, les Studie d'Oskar Fischinger (1929-1932), etc. La conception de ces ensembles filmiques est tributaire d'un double principe: d'une part, ils reposent parfois sur des modèles structurels et compositionnels empruntés à des arts préexistants, essentiellement la peinture et la musique. D'autre part, ils reposent sur une connaissance approfondie des propriétés du médium, que les cinéastes explorent dans une démarche réflexive, en particulier celle qui concerne la reproductibilité technique de l'image cinématographique et le caractère fondamentalement répétitif de la bande filmique, agencée en photogrammes successifs et équidistants.

Dans la mesure où les séries télévisées sont si souvent présentées comme des formes qui se réapproprient ou qui prolongent des questions posées dans le champ du cinéma, on peut donc s'étonner qu'il n'existe - à notre connaissance - aucune tentative de ce type dans l'histoire des formes sérielles télévisuelles, où la narrativité règne à peu près sans partage. Faut-il comprendre par-là que les séries télévisées seraient condamnées à la forme narrative-représentative ? Que la narration serait à tel point chevillée au corps de la sérialité télévisuelle que le média serait, au fond, incapable de prendre en charge et 
d'amplifier les échos de ce qui a pourtant et si profondément agité la sphère des arts plastiques, ou celle de la musique, au cours du XIX ${ }^{e}$ siècle et tout au long du suivant? Pourtant, à l'observer du point de vue élargi d'une histoire des formes, il nous semble que la sérialité est à la fois distincte du modèle narratif - qu'elle peut cependant recouper -, et, parfois, liée plus frontalement à l'ambition d'une déprise de ce modèle. C'est ce dont attestent diversement les variations à l'œuvre dans l'histoire de la gravure, l'ensemble formé par les quelque 250 tableaux composant les Nymphéas de Claude Monet, les sérigraphies d'Andy Warhol ; mais aussi les compositions atonales de la musique sérielle chez Schönberg ou Webern, les Cent mille milliards de poèmes ou les Exercices de style de Raymond Queneau, ou le principe du sampling dans les musiques dites " actuelles", pour donner des exemples bien différents mais qui partagent une conception ouverte de la sérialité, où la narrativité est au mieux un effet collatéral. L'approche exclusivement narrative de la sérialité fait ainsi écran, nous semble-t-il, aux dialogues potentiellement fructueux que les séries télévisées pourraient entretenir non seulement avec d'autres sphères de la création artistique, mais aussi avec les modèles compositionnels ou structuraux dont elles procèdent, et dont elles se nourrissent, quant à elles, allègrement. Pour ne prendre qu'un seul exemple, la dimension collective, voire chorale, d'une série telle que The Walking Dead (AMC, 2010-), ne ressortit pas uniquement à des impératifs de narrativité. La manière dont elle organise les rapports spatiaux de ses protagonistes en une forme de feuilletage permanent, indique certes une volonté de problématiser la figure du leader, et d'approfondir successivement la peinture des caractères de la communauté des personnages; mais elle thématise aussi des stratégies d'écriture collective, celles de la "writer's room», auxquelles elle renvoie de façon réflexive. Elle organise ses éléments en fonction d'un principe de polyphonie. Elle invente des modèles d'interaction qui peuvent susciter l'intérêt hors de toute considération sur le devenir de cette communauté dans un monde en ruines. Elle bâtit des architectures relationnelles qui affectent aussi son esthétique, à travers les valeurs du cadre, les interpolations du montage, le traitement des rapports entre figure et fond, ou entre surface et profondeur.

Dans un article publié en 2008, Olivier Aïm faisait l'hypothèse d'une « écriture tabulaire ${ }^{47}$ » à l'œuvre dans la sérialité télévisuelle, permettant, peut-être, de sortir de l'ornière narrative. L'idée d'une lecture tabulaire, opposée à la lecture linéaire, fut également l'un des objets d'étude des chercheurs du Groupe $\mu(\mathrm{Mu})$ dans leur tentative de conceptualisation de la rhétorique poétique, à la fin des années $1970^{48}$. À la fois plus près et plus loin de nous, la notion de tabularité fut aussi mise en avant dans les travaux consacrés par Georges Didi-Huberman ou Philippe-Alain Michaud à l'historien d'art Aby Warburg. L'Atlas Mnémosyne, que ce dernier s'était employé à édifier au cours des années 1920, constitua pour bien des chercheurs un outil permettant de penser le devenir des images, leurs modes de "survivance» et de circulation, les vertus heuristiques de l'anachronisme, du déplacement et du montage. La tabularité de l'atlas, fait de hiatus et de bifurcations, propose ainsi une alternative à la linéarité supposée par le modèle de l' istoria, telle qu'a pu la mettre en avant Erwin Panofsky, qui était pourtant l'un des disciples de Warburg ${ }^{49}$. Bien entendu, une pensée «tabulaire » des images n'implique pas l'abandon de tout lien avec la narrativité. Mais elle suggère de mettre l'accent à la fois sur le rôle de la plasticité des formes et sur des effets de structure qui, sans faire disparaître le récit, le dispersent suffisamment pour laisser le regard libre d'arpenter les images en fonction d'autres logiques. Mettre la narrativité « en mineur » (pour continuer de filer la métaphore musicale) dans les formes sérielles, ce serait donc, aussi, permettre à différents modèles épistémiques de se faire jour dans leur analyse. De déployer ou 
d'étoiler les formes, aussi bien que l'expérience que nous en faisons, et la façon dont nous en rendons compte.

Envisager d'autres modèles pour l'analyse de la sérialité télévisuelle implique ainsi d'être attentif à l'historicité des pratiques sérielles, et à la diversité des formes qu'elles empruntent dans le contexte contemporain. À défaut d'une véritable typologie, nous voudrions donc indiquer au moins deux grandes voies possibles, qui permettraient de concevoir des modes d'analyse des productions sérielles télévisées présentant une alternative - ou un complément - au paradigme narratif. La première viserait à considérer ce qui s'élabore, dans les séries, au niveau structural, en substituant au modèle sémantique dominant un modèle davantage syntaxique, quitte à délaisser l'analyse des œuvres et à adopter des cadres d'interprétation quantitatifs, à grande échelle, qui permettraient de valoriser l'accumulation de données plutôt que des modèles d'organisation linéaires. La seconde voie consisterait inversement - et sans que ce soit contradictoire avec le modèle structural - à évaluer ce que serait l'apport d'une approche plus résolument formaliste des séries télévisées, qui ont été singulièrement peu observées à l'aune de la plasticité qu'elles manifestent. Dans cette optique, il s'agirait notamment de dégager les vertus d'une analyse qui ne prendrait pas pour cadre de référence le média télévisuel, mais bien plutôt le médium - film, vidéo analogique ou numérique -, et les puissances expressives qu'il recèle.

Ainsi, une première piste de réflexion consisterait à s'intéresser à la dimension structurelle des séries télévisées à partir d'autres formes artistiques, en veillant à ne pas assujettir la structure à la narration. Il nous semble en effet qu'une distinction entre deux types de sérialité télévisuelle peut être envisagée a minima. D'un côté, certaines séries ( Breaking Bad ${ }^{50}$, Lost, The Wire ${ }^{51}$, The Walking Dead, Games of Thrones ${ }^{52}$, etc.), faisant actuellement l'objet d'une large reconnaissance pour leur grande complexité narrative, déploient une approche spatiale relevant d'un modèle expansif. Ces séries tournent en effet autour de l'imaginaire de la frontière, chaque saison ayant pour objectif son dépassement et la découverte de nouveaux espaces, sur un modèle qui rappelle par bien des aspects celui du western - mais aussi certains modèles cartographiques développés dans le cadre du jeu vidéo. De l'autre, ces séries à cadre fixe que sont la sitcom ou le soap opera relèvent d'une approche spatiale différente, intensive, qui vise l'épuisement d'un espace unique et souvent fermé (appartement, immeuble, bureaux d'entreprise, école, etc.). Elles fonctionnent à partir de décors invariants, lesquels contraignent davantage à une exploration des potentialités spatiales qui fonctionnerait par superposition et accumulation. L'étude de cette modalité de la spatialité sérielle, relativement peu menée jusqu'ici, permettrait d'envisager comment des contraintes liées à la production engendrent des formes innovantes rappelant moins les recherches du roman-feuilleton que celles, par exemple, de l'Oulipo. Fondé au début des années 1960 autour de Raymond Queneau et du mathématicien François Le Lionnais, l'OUvroir de Littérature POtentielle constitue un groupe de recherche œuvrant à produire de nouvelles structures et formes littéraires à partir de procédures contraignantes recherchées, travaillées et assumées. Convaincus que les contraintes forment un puissant levier d'investigation des potentialités littéraires, ses membres composent à partir de structures mathématiques, musicales ou encore ludiques. S'il n'existe pas (encore) d'ouvroir pour la série télévisée, les contraintes de production, lorsqu'elles sont assumées comme de potentiels outils de création et non destinées à être dissimulées le plus possible au spectateur avec la complicité de la narration, fournissent de beaux exemples d'approches liées à la question 
de l'habitation d'un espace. L'épuisement des possibilités offertes par un lieu mais également par un médium constitue un enjeu créatif novateur évoquant la Tentative d'épuisement d'un lieu parisien ou La vie mode d'emploi de Georges Perec ${ }^{53}$. Une telle perspective pourrait bénéficier par exemple de modèles d'analyse quantitatifs tels que ceux utilisés par Franco Moretti pour la littérature, lequel s'attache dans Graphes, cartes et arbres $^{54}$ à une histoire quantitative du genre romanesque et de ses sous-genres dans le monde entier. Ce type d'approche à très grande échelle pourrait être transposé dans l'analyse de ces constellations de séquences, d'épisodes, d'arcs narratifs et de saisons, laissant de côté leur construction linéaire pour envisager des structures fondées sur ces éléments essentiels que sont l'accumulation et la réitération.

Figure 1 : Guillaume Duchenne de Boulogne, Mécanisme de la physionomie humaine ou Analyse électro-physiologie de l'expression des passions, 1862, planche 7
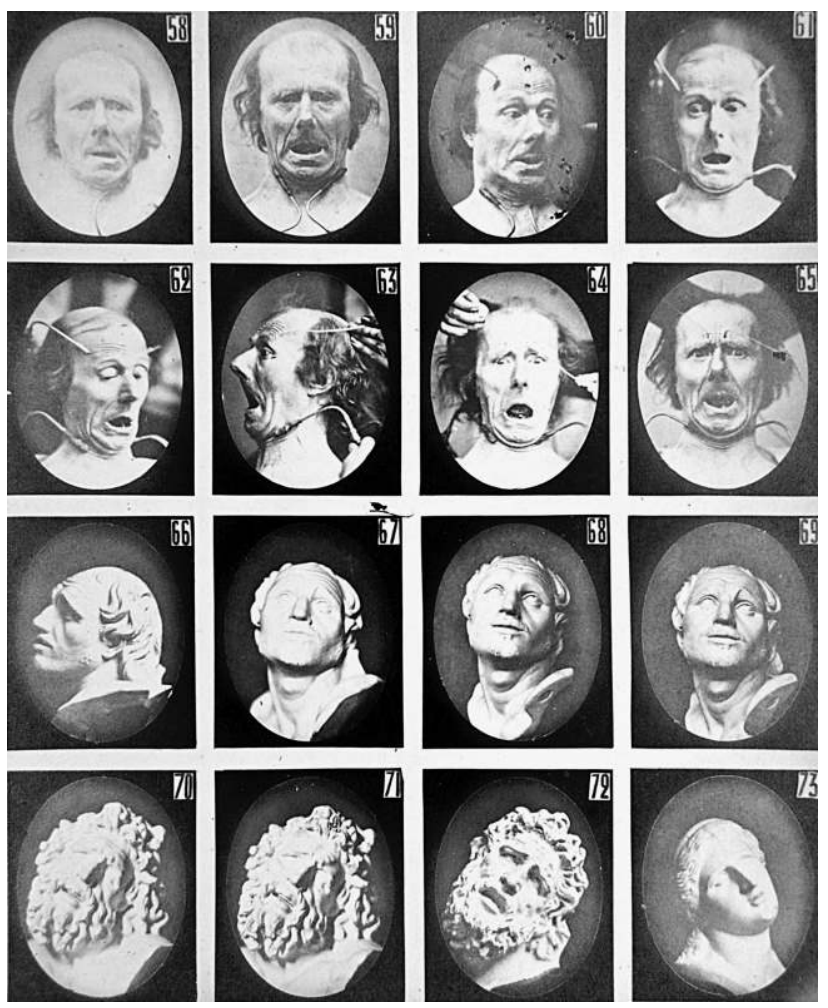

Que peut un espace sériel? Que peut un corps, pris dans un tel espace? Travailler ces enjeux nécessite l'adoption d'un regard attentif à la dimension de répétition et de variation des motifs structuraux des séries télévisées. Les répétitions de grimaces, mimiques, cris, répliques, gestes de Sheldon Cooper dans The Big Bang Theory (CBS, 2007-2019), pour ne prendre que cet exemple, sont autant de variations burlesques autour d'un motif, variations bien peu soumises à la linéarité temporelle de la même façon que les planches photographiques de Guillaume Duchenne de Boulogne au mitan du XIX siècle, qui documentaient ses expériences sur le traitement clinique par l'électricité, recourant à des formes de montage sériel qui mettaient en regard les mimiques de ses patients avec les formes du pathos développées par la statuaire grecque (Fig. 1). À l'instar du roman Marelle de l'écrivain argentin Julio Cortazar ${ }^{55}$, plusieurs modes de visionnage des séries télévisées sont ainsi possibles. Si le téléspectateur peut évidemment prendre plaisir dans le fait de suivre l'ordre narratif prévu par les créateurs d'une série, il peut tout aussi bien visionner des épisodes dans un autre ordre, choisi («montage» électif 
d'épisodes) ou subies ${ }^{56}$. Le jeu de variation peut également résulter de stratégies économiques visant à adapter une série à succès d'un pays à un autre. Ainsi, les scénaristes et réalisateurs de l'adaptation franco-belge de la teen-série norvégienne Skam (NRK, 2015-2017) se sont vus contraints par contrat de suivre scrupuleusement pour les premiers épisodes les éléments de mise en récit et de mise en scène de la série originale (dialogues, échelles de plan, costumes, etc.). Au-delà des traditionnels enjeux de remake et d'adaptation, la simple possibilité de reconduction d'une image en mouvement à travers d'autres corps et d'autres espaces conduit à se demander ce qui fait la singularité d'une présence, ou d'une image, chaque plan constituant une attraction pour le spectateur familier de la version norvégienne, produisant chez lui un regard étonné et attentif, un regard curieux non tant en termes de narration que de variations autour d'un référent, d'un modèle. Pour questionner ces jeux d'échos, de reprises et variations, des approches extérieures à la narratologie sont à explorer en ce qu'elles apportent des potentialités d'analyse particulièrement fécondes. Ces approches visant à questionner les formes sérielles autant à partir des pratiques spectatorielles que des impératifs de production invitent donc à de nouvelles interrogations et possibilités d'analyse... mais elles obligent, pour ce faire, à convoquer des champs de la création ou du savoir dans lesquels la narrativité n'est pas, ou plus, un enjeu princeps.

Figure 2 : La Linea, épisode 9, 1978 (RAI)

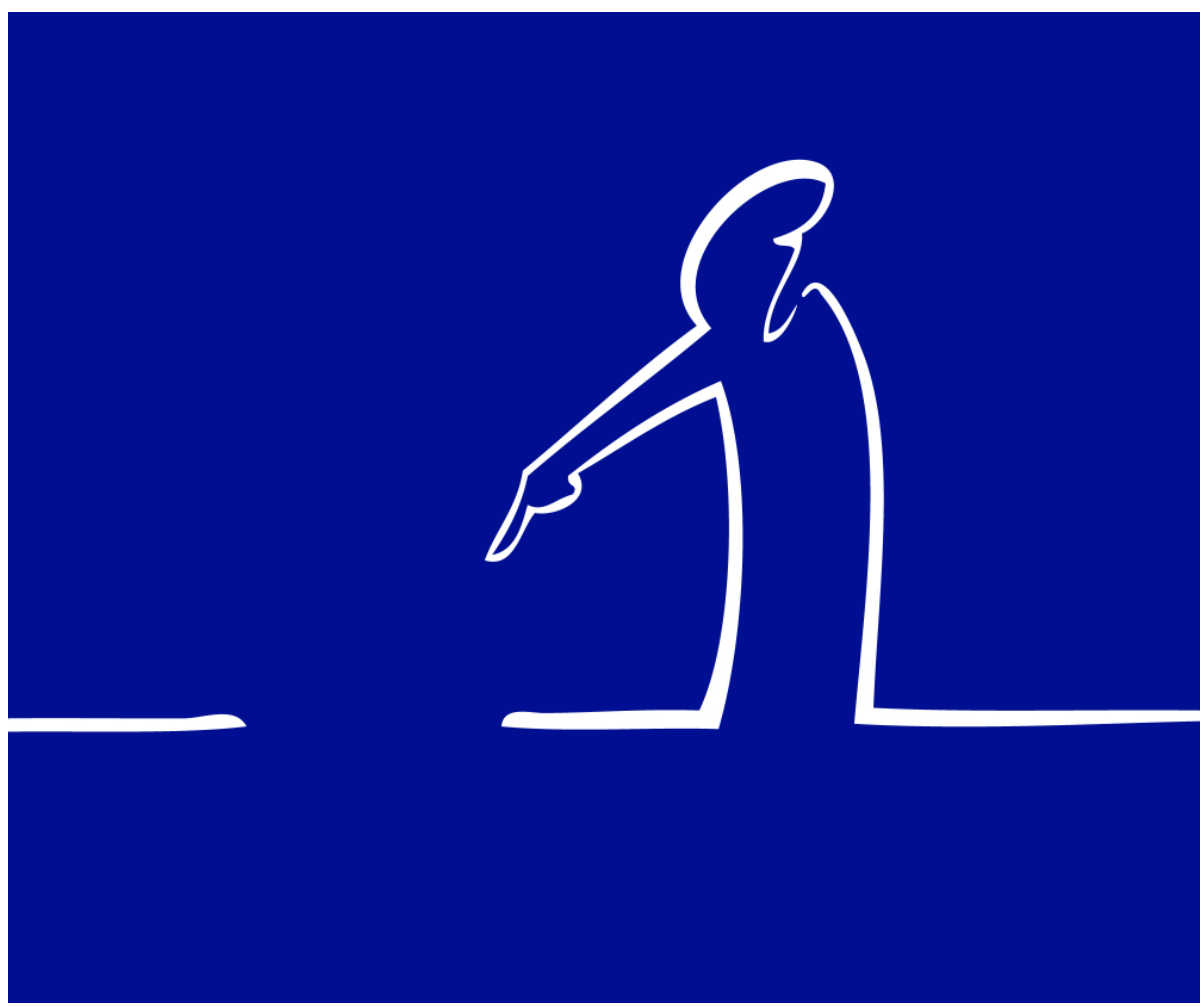

À l'autre bout du spectre analytique, les nombreuses réflexions autour de la plasticité des images et de la spécificité du médium pourraient aussi constituer des propositions pour envisager l'étude de la sérialité télévisuelle. Il est étonnant par exemple que le stimulant ouvrage de Dominique Avron Le scintillant ${ }^{57}$ n'ait pas fait plus d'émules dans l'analyse des formes télévisuelles. Or, les séries télévisées peuvent consciemment jouer (et se jouer) avec les paramètres techniques et esthétiques qui permettent la fabrication d'une image de télévision, cathodique ou numérique. Les 90 épisodes de la série d'animation italienne 
La Linea, diffusée sur la RAI puis un peu partout dans le monde à partir de 1971, ne racontent pas autre chose que les tours et les détours, les circonvolutions et les balayages d'une ligne, qui renvoie aussi à la trame télévisuelle, ce métier à tisser sur lequel étaient fabriquées les formes cathodiques (Fig. 2). Plus près de nous, dans "The Signal » (S04E24), un épisode du dessin animé The Amazing World of Gumball (Cartoon Network, 2011-), le visage d'un personnage peut tout à coup se décomposer en une succession de pixels, le son devenir saccadé. Le téléspectateur peut alors supposer une défaillance électronique momentanée de son téléviseur, mais ce glitch, cette erreur visuelle qui est aussi une forme à part entière, est en réalité un élément prévu dans la diffusion, jetant le trouble sur l'origine de cet incident et renvoyant la série à sa dimension autoréflexive (Fig. 3-5).

Figure 3 : The Amazing World of Gumball, S04E24, « The Signal »

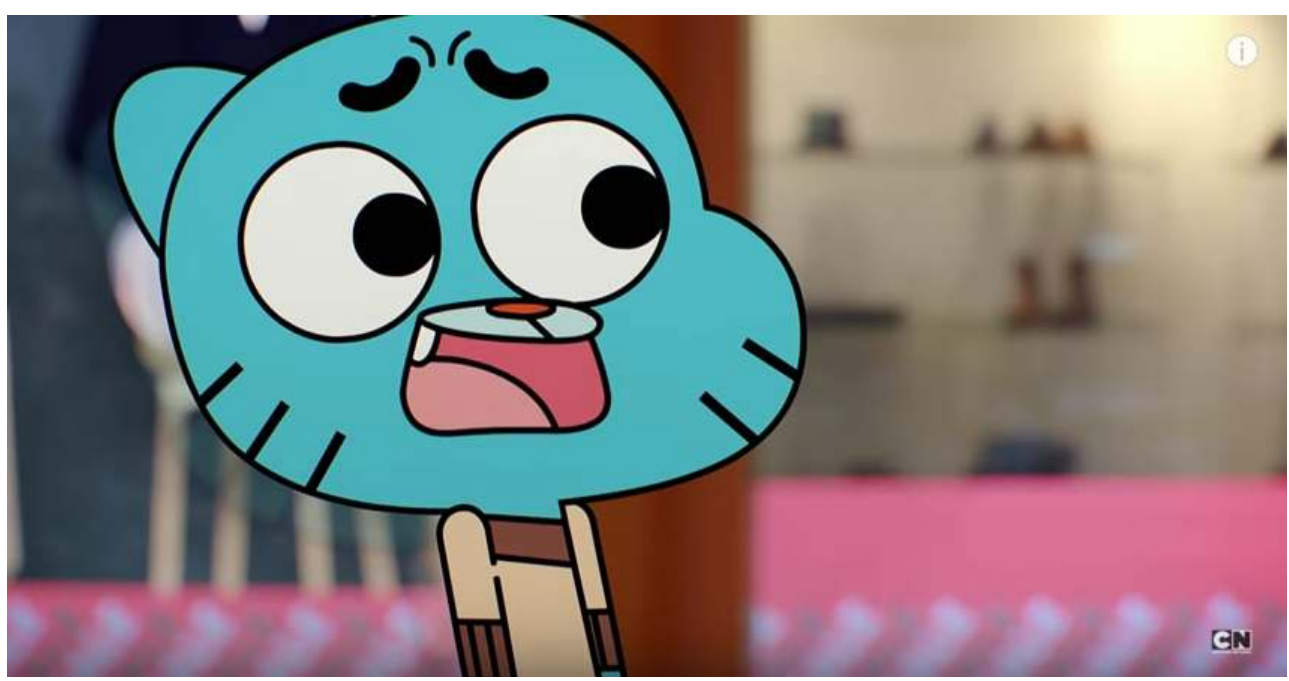

Figure 4 : The Amazing World of Gumball, S04E24, « The Signal »

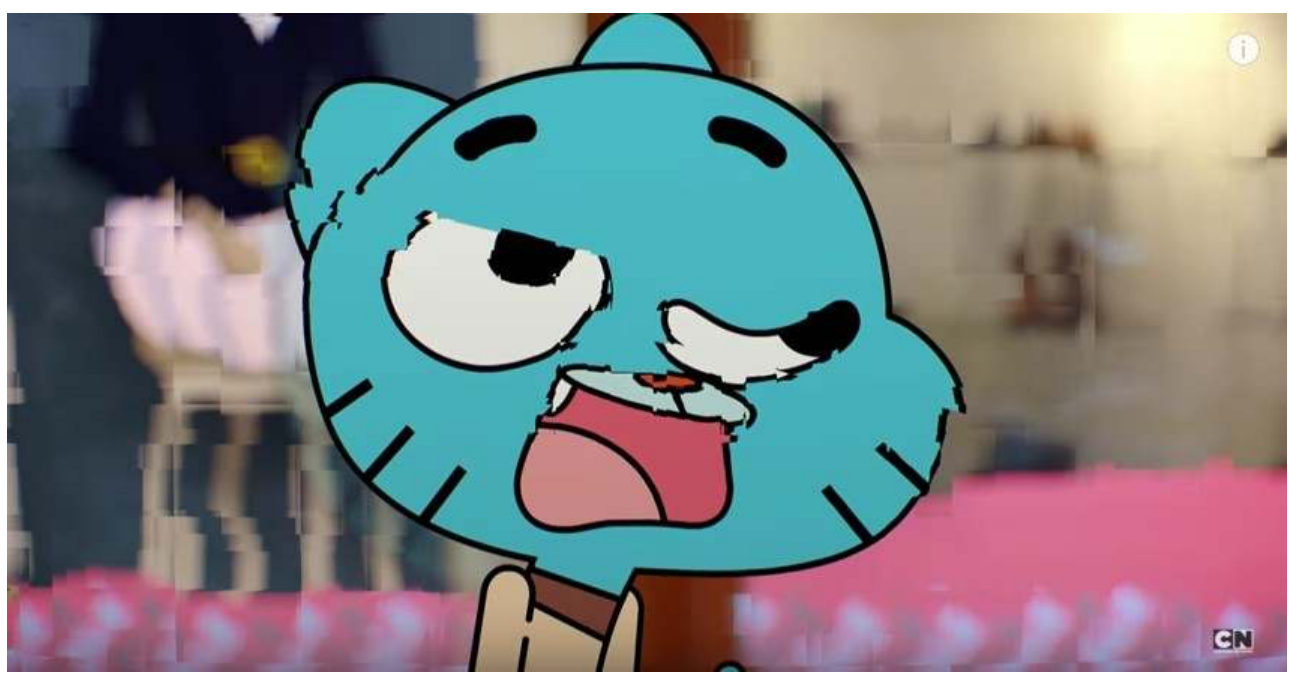


Figure 5 : The Amazing World of Gumball, S04E24, « The Signal »

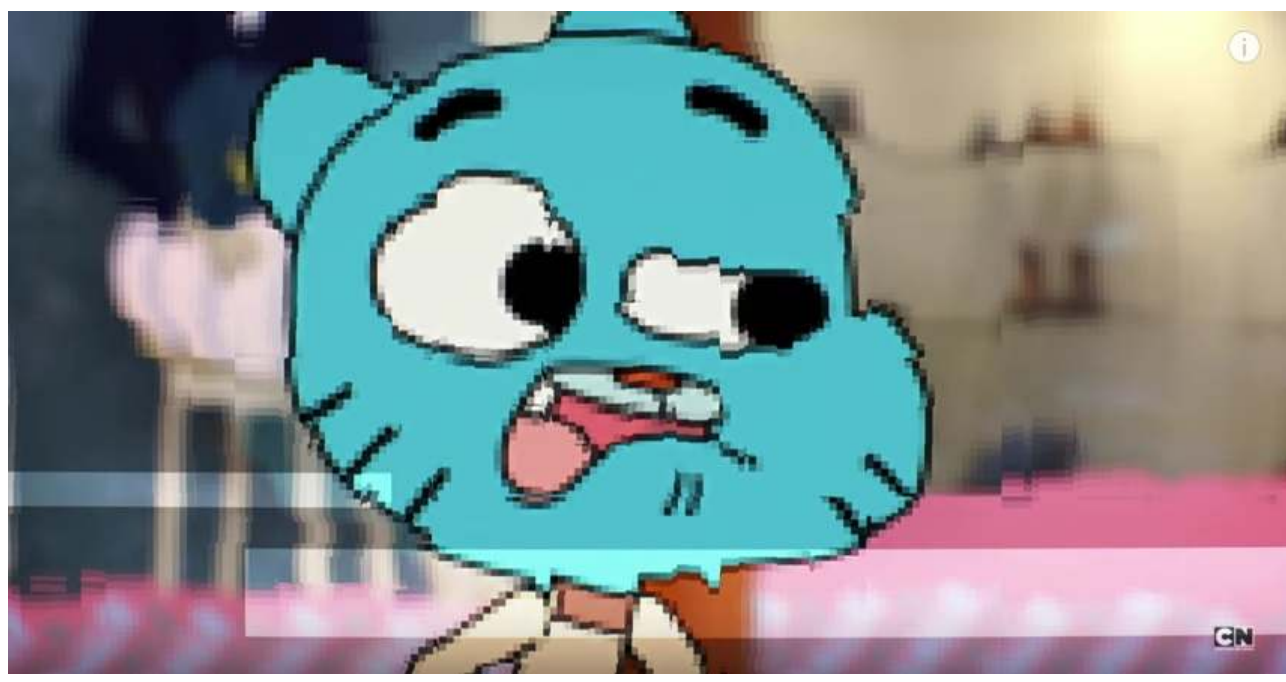

Certaines séries vont plus loin encore, comme le dessin animé Adventure Time (Cartoon Network, 2010-2018) dont un épisode intitulé «A Glitch is a Glitch» (S05E15) est entièrement construit autour d'une perturbation du flux numérique qui n'affecte pas seulement les conditions de réception du dessin animé, mais contamine - autant qu'il structure - la mise en récit elle-même (Fig. 6-9). Que ces exemples soient pris dans le champ de l'animation ne doit pas surprendre, tant les libertés prises avec le principe même de représentation y sont plus grandes que dans les séries en prises de vues réelles. Mais dans ces dernières, une fois encore, la narrativité est-elle une fatalité ?

Figure 6 : Adventure Time, S05E15, «A Glitch is a Glitch »

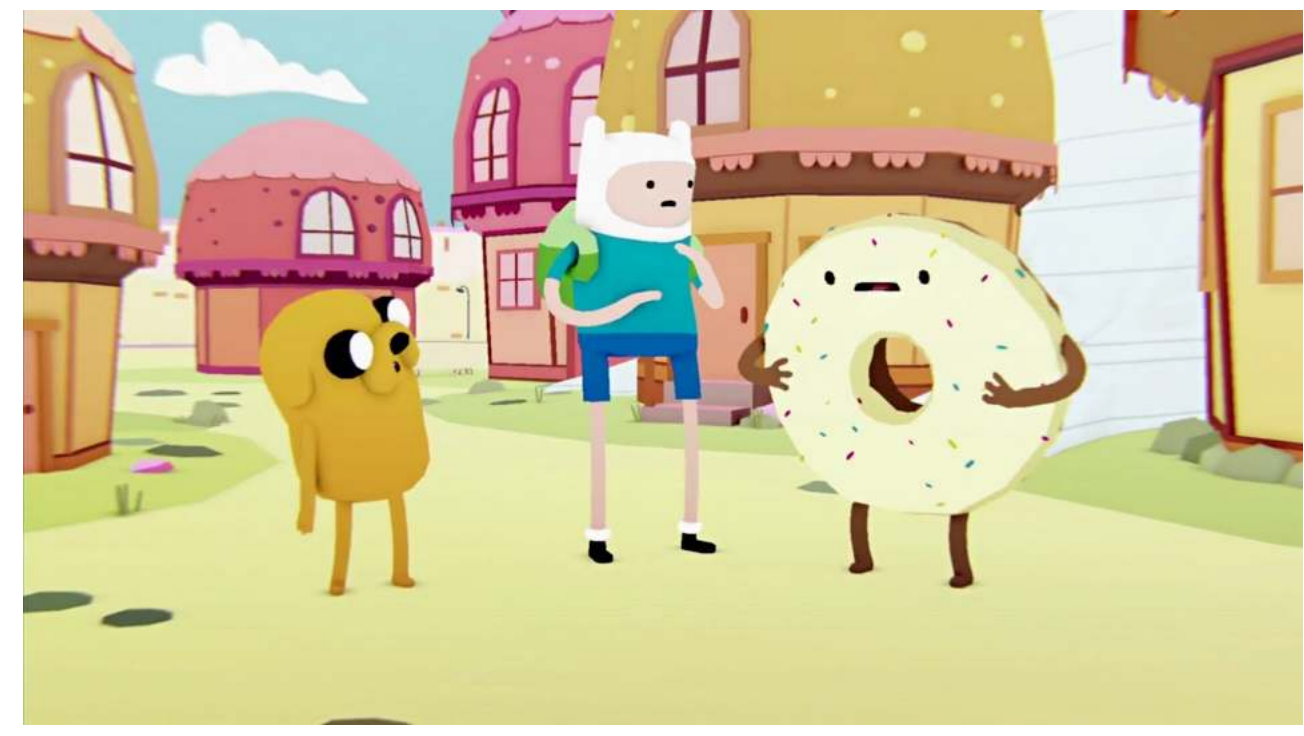


Figure 7 : Adventure Time, S05E15, «A Glitch is a Glitch »

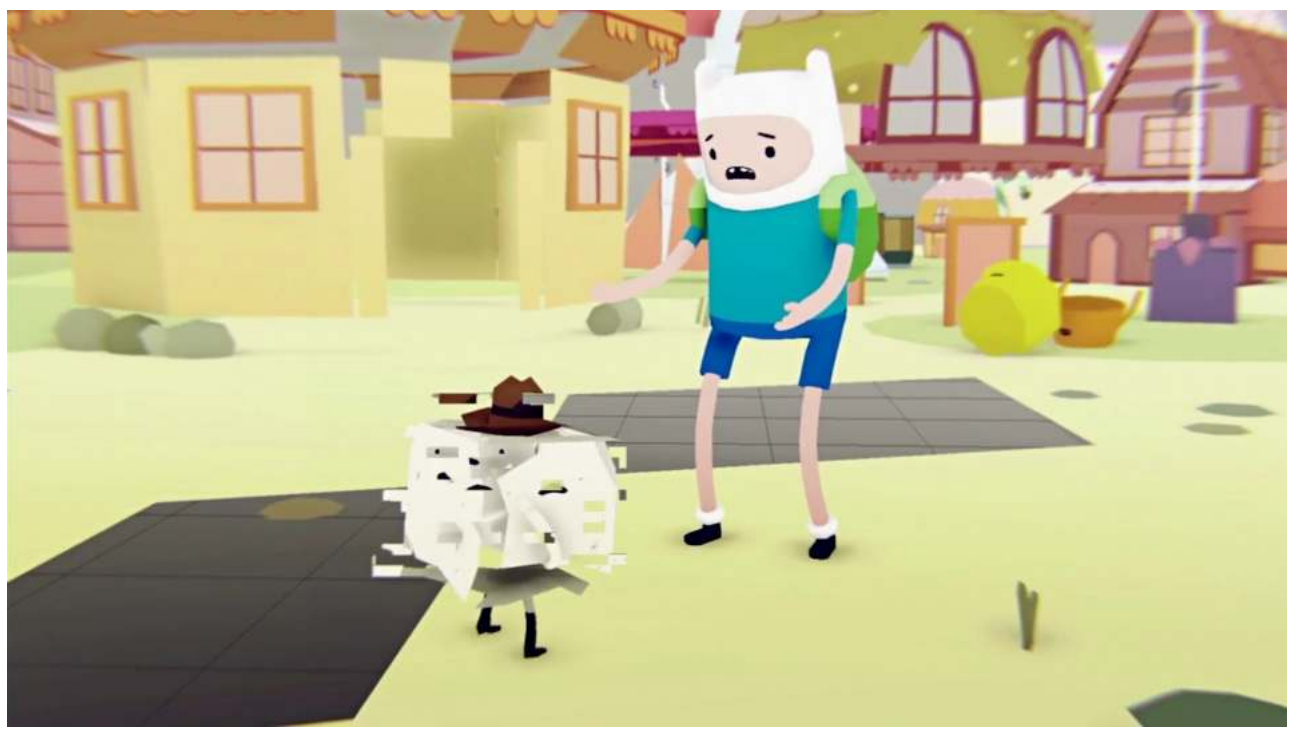

Figure 8 : Adventure Time, S05E15, «A Glitch is a Glitch »

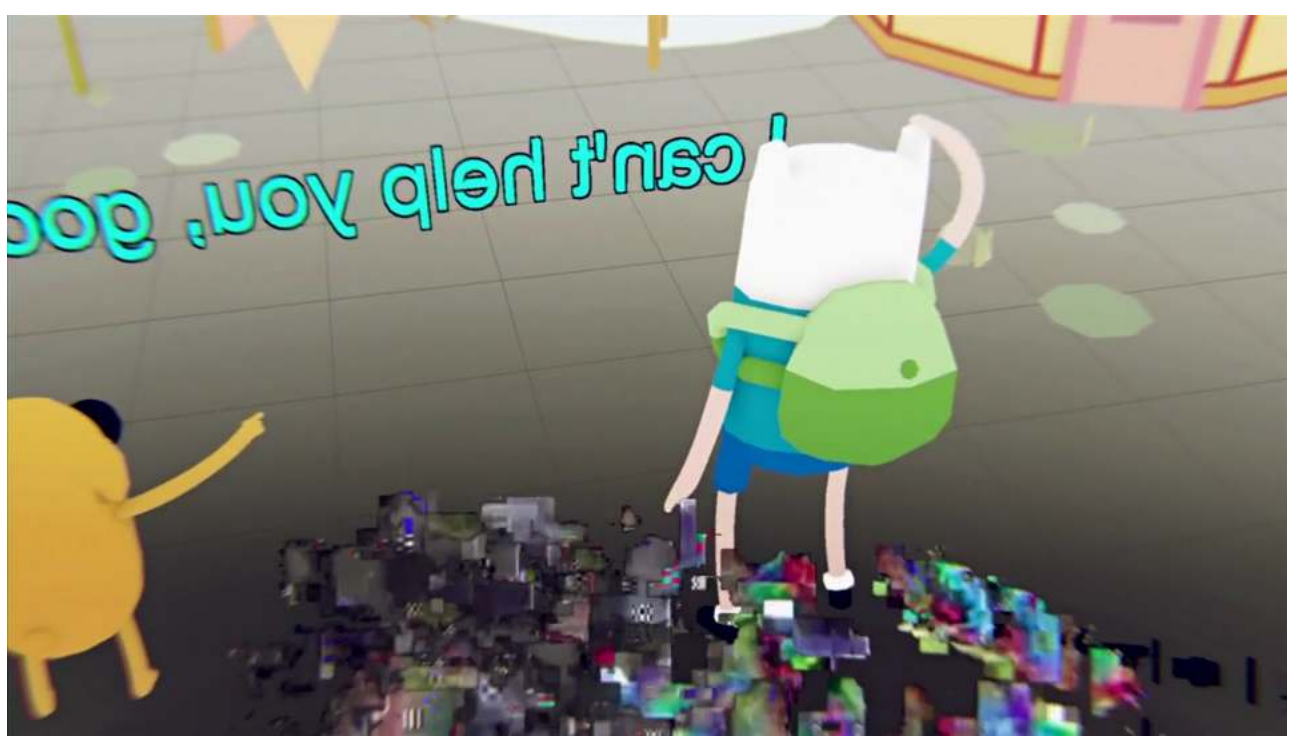


Figure 9 : Adventure Time, S05E15, «A Glitch is a Glitch »

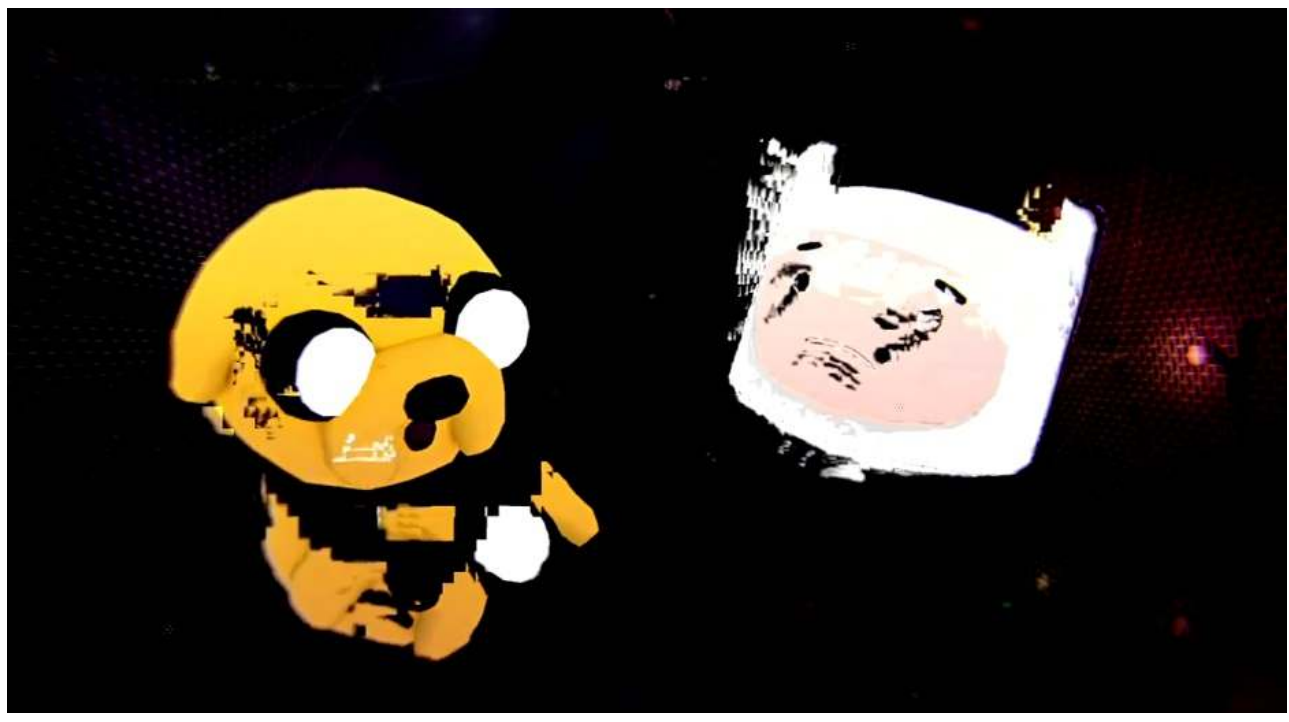

31 L'apparition de ces images à double fond, qui interrogent la condition même des séries à l'ère numérique, produit ainsi non seulement des représentations, mais aussi des questionnements d'un nouveau type, en interrogeant «l'interdépendance entre le développement technique et son insertion sociale et culturelle ${ }^{58}$.» Ces pratiques des images ne renvoient pas uniquement à ce qu'on qualifie parfois de séries "méta ", qui mettent en abîme leur propre condition de récits audiovisuels ${ }^{59}$. Celles et ceux qui fabriquent les séries connaissent les enjeux techniques d'une image numérique, de sa production et de sa transmission, et il est donc curieux que cette dimension n'ait pas été davantage relevée par les commentateurs des séries, faute peut-être d'outils appropriés pour s'emparer de ces questions.

Il nous semble donc, pour conclure, que les études télévisuelles gagneraient à contester plus systématiquement le postulat selon lequel une série télévisée est d'abord et toujours un récit, indexé sur des effets de ressemblance avec le monde qui la produit.

Que peut une image? Faute de se poser plus frontalement cette question, qui agite pourtant le cinéma comme l'ensemble des arts visuels depuis longtemps, les études télévisuelles restent pour ainsi dire en deçà de ce qu'elles pourraient accomplir. On l'a dit, il ne s'agit pas d'évaluer les séries à partir d'un critère comme celui de la mise en scène, et moins encore de suggérer que tout reste à faire, ou que rien n'a été fait, loin de là. Mais plutôt de plaider pour une conception de la sérialité qui soit moins unilatéralement sémantique, c'est-à-dire à la fois plus spécifique - en évaluant les formes télévisuelles à l'aune de leur plasticité propre - et plus ouverte - en restant attentif à d'autres conceptions de la création sérielle, qui ont informé d'autres pans de l'activité artistique, en d'autres époques, et continuent de le faire.

Ce texte n'avait pas pour ambition d'apporter les réponses à un tel problème, mais, plus simplement, de pointer son existence. Il faut donc le prendre, avec modestie, pour ce qu'il est : un chantier, fait de constats et de propositions, un travail à faire, en devenir, et une invitation lancée aux chercheuses et chercheurs afin de les inciter à faire confiance aux formes, qui sont toujours plus libres, plus inventives, plus stimulantes que ce à quoi l'industrie qui les fabrique voudrait les réduire (des histoires). Regarder ailleurs que là où on nous dit de le faire, c'est déjà regarder autrement. 


\section{BIBLIOGRAPHIE}

AÏM, Olivier, « La série télévisée comme machine à voir ", Entrelacs, Hors-série « Séries télé in/ out », 2008, mis en ligne le 1er août 2012 : http://entrelacs.revues.org/260

ANONYME, «Éclair - Nick Carter, Roi des détectives », Ciné-Journal, n5, 15 septembre 1908, p. 4.

ANONYME, «"Give Them What They Don't Expect" - Feuillade ", Motion Picture News, Volume 14, n²0, 18 novembre 1916, p. 3163.

ANONYME, « Lone Thief Appears and Opposes "The Vampires" in Third Episode ", Motion Picture News, Volume 14, n²2, 2 décembre 1916, p. 3490.

ANONYME, «Observations philatéliques », Revue philatélique française, n85, décembre 1897.

ANONYME, « Serial », The Oxford English Dictionary, Oxford, Clarendon Press, (1933) 1961.

ANONYME, « Série ou Suite : en Algèbre », dans Diderot, D’Alembert, L'Encyclopédie, Volume 15, 1765, p. 93b-96a : http://enccre.academie-sciences.fr/encyclopedie/article/v15-174-0

ARISTOTE, Poétique, traduit par Barbara Gernez, Paris, Les Belles Lettres, coll. « Classiques en poche », (2001) 2008.

AVRON, Dominique, Le scintillant - Essai sur le phénomène télévisuel, Strasbourg, Presses universitaires de Strasbourg, 1994.

BARONI, Raphaël, JOST, François (dir.), Télévision, $n^{\circ} 7$, « Repenser le récit avec les séries », CNRS, 2016.

BENASSI, Stéphane, Séries et feuilletons TV - Pour une typologie des fictions télévisuelles, Liège, CÉFAL, coll. «Grand Écran Petit Écran », 2000.

BESSON, Anne, Constellations. Des mondes fictionnels dans l'imaginaire contemporain, Paris, CNRS, 2015.

BREDA, Hélène, « Le « Tissage narratif » et ses enjeux socio-culturels dans les séries télévisées américaines contemporaines », thèse de doctorat sous la direction de Guillaume Soulez, Université Paris 3 - Sorbonne-Nouvelle, 2015.

CICÉRON, De la divination, Paris, Librairie Garnier Frères, coll. « Classiques Garnier », 1937. DIDI-HUBERMAN, George, L'image survivante - Histoire de l'art et temps des fantômes selon Aby Warburg, Paris, Minuit, coll. « Paradoxes », 2002.

ESQUENAZI, Jean-Pierre, Les Séries télévisées - L'avenir du cinéma ?, Paris, Armand Colin, 2014. ESQUENAZI, Jean-Pierre, Éléments pour l'analyse des séries, Paris, L'Harmattan, coll. « Champs visuels », 2017.

GAUDREAULT, André, JOST, François, Le Récit cinématographique, Paris, Armand Colin, (1990) 2017. GUÉROULT, Constant, « Du roman-feuilleton », La Presse dramatique, n¹9, 28 décembre 1851. GROENSTEEN, Thierry, « Fictions sans frontières », in GAUDREAULT, André, GROENSTEEN, Thierry, La Transécriture. Pour une théorie de l'adaptation, Québec, Nota Bene, 1998. 
GROUPE $\mu$, Rhétorique de la poésie - Lecture linéaire, lecture tabulaire, Bruxelles, Éditions Complexe, 1977.

JOST, François, Comprendre la télévision et ses programmes, Paris, Armand Colin, coll. « Focus

Cinéma », (2005) 2017.

LANCIEN, Thierry, «Un objet improbable », MédiaMorphoses, $\mathrm{n}^{\circ} 6$, « La télévision : média de la parole?», novembre 2002.

LECOINTE, Thierry, « Les premières années du spectacle cinématographique à Nîmes 1895-1913», 1895, n43, été 2004, p. 57.

LETOURNEUX, Matthieu, Fictions à la chaîne : littératures populaires et culture médiatique, Paris Seuil, coll. « Poétique », 2017.

MAUCLAIR, Camille, L'Impressionnisme, son histoire, son esthétique, ses maîtres, Paris, Librairie de l'Art ancien et moderne, 1904.

MICHAUD, Philippe-Alain, Aby Warburg et l'image en mouvement, Paris, Macula, (1998) 2012.

MITTELL, Jason, Complex TV : The Poetics of Contemporary Television Storytelling, New York, NYU Press, 2015.

MORETTI, Franco, Graphes, cartes et arbres - Modèles abstraits pour une autre histoire de la littérature, Paris, Les Prairies ordinaires, coll. «Penser/Croiser », 2008.

PANOFSKY, Erwin, Essais d'iconologie - Thèmes humanistes dans l'art de la Renaissance, Paris, Gallimard, (1939) 1967.

PEREC, Georges, Tentative d'épuisement d'un lieu parisien, Paris, UGE, 1975.

PEREC, Georges, La vie mode d'emploi. Romans, Paris, Hachette, 1978.

PLINE L'ANCIEN, Histoire naturelle, Livre XI, Paris, Les Belles Lettres, « collection des universités de France ", 1947.

QUINTE-CURCE, Histoires, Paris, Les Belles Lettres, « Collection des universités de France », (1948) 1965.

QUINTILIEN, Institution oratoire, Paris, Librairie Garnier Frères, coll. « Classiques Garnier », 1933.

REY, Alain (dir.), Dictionnaire historique de la langue française, Paris, Dictionnaires Le Robert, (1993) 2010.

SEPULCHRE, Sarah, « Le héros multiple dans les fictions télévisuelles à épisodes :

complexification du système des personnages et dilution des caractérisations ", thèse de doctorat en sciences sociales, sous la direction de Marc Lits, Louvain, UCL, 2007.

STACE, Silves, Paris, Les Belles Lettres, « Collection des universités de France », 1944.

THOMPSON Robert J., Television's Second Golden Age, Syracuse, Syracuse University Press, 1997.

\section{NOTES}

1. Le texte a été écrit par Sophie Barel, Quentin Fischer, Jean Gueguen, Damien Keller, Yannick Kernec'h, Jean-Baptiste Massuet et Éric Thouvenel.

2. Robert J. Thompson, Television's Second Golden Age, Syracuse, Syracuse University Press, 1997.

3. Jason Mittell, Complex TV: The Poetics of Contemporary Television Storytelling, New York, NYU Press, 2015. 
4. Thierry Groensteen, « Fictions sans frontières », dans André Gaudreault et Thierry Groensteen, La Transécriture. Pour une théorie de l'adaptation, Québec, Nota Bene, 1998, p. 14.

5. Jean-Pierre Esquenazi parle également de «l'art (narratif) des séries télévisées ». Jean-Pierre Esquenazi, Les Séries télévisées - L'avenir du cinéma ?, Paris, Armand Colin, 2014.

6. Thierry Groensteen, « Fictions sans frontières », art. cit., p. 15.

7. «Il est dans la nature de tout récit de focaliser l'attention du public sur son déroulement fictionnel et d'occulter, dans une certaine mesure, le travail accompli par l'artiste sur les signes à travers lesquels le récit se manifeste. [...] cette transparence relative de la forme n'est nullement un effet spécifique à la littérature ; elle s'observe (avec des nuances) dans tous les arts narratifs. Elle est le résultat de l'impérialisme intrinsèque de la forme récit qui, toujours, récupère à son profit l'ensemble des éléments composant l'œuvre. Comme le dit Christian Metz: "Dans un film narratif, tout devient narratif, même le grain de la pellicule ou le timbre des voix". Bref, un film, un roman ou une BD sont essentiellement, pour un public avide d'histoires et aiguillonné par la curiosité, la manifestation concrète d'un scénario, son corps phénoménologique. », Groensteen., p. 17-18.

8. Stéphane Benassi, Séries et feuilletons TV - Pour une typologie des fictions télévisuelles, Liège, CÉFAL, coll. « Grand Écran Petit Écran », 2000.

9. Ce processus de légitimation face à une culture du préjugé ressemble fort à celui qu'ont connu successivement le roman et le cinéma. Cf. François Jost, Comprendre la télévision et ses programmes, Paris, Armand Colin, coll. « Focus Cinéma », (2005) 2017, p. 17-18.

10. Voir Jean-Pierre Esquenazi, Éléments pour l'analyse des séries, Paris, L'Harmattan, coll. «Champs visuels ", 2017, p. 7.

11. François Jost et André Gaudreault, par exemple, ont publié en 2017 une troisième édition, revue et augmentée, de l'ouvrage Le Récit cinématographique, qui renouvelle le corpus d'origine pour y intégrer des analyses de séries télévisées. Les auteurs y défendent l'idée que " "récit cinématographique" peut faire référence à la fois aux films et aux séries ", mais admettent que « récit audiovisuel» serait plus moderne. André Gaudreault et François Jost, Le Récit cinématographique, Paris, Armand Colin, (1990) 2017, p. 12-13.

12. Hélène Breda, «Le "tissage narratif" et ses enjeux socioculturels dans les séries télévisées américaines contemporaines", thèse de doctorat en études cinématographiques et audiovisuelles, sous la direction de Guillaume Soulez, Paris, Université Sorbonne-Nouvelle Paris 3, 2015.

13. Respectivement : BBC One, 1963-1989 et 2005-- ; Netflix, 2016-2017 ; Channel 4, 2004-2013 ; NBC, 2005-2013 ; NBC, 2009-2014 et Yahoo Screens, 2015.

14. Nous indiquons "fictionnelle" dans la mesure où cette relation est avant tout le produit d'une activité de l'imaginaire du spectateur, ne donnant pas lieu à un échange véritable. Toutefois les nombreuses questions que cette idée soulève, particulièrement dans un contexte artistique contemporain valorisant l'échange dynamique entre création et réception (ce que la télévision a en un sens anticipé et accompagné), nous poussent à l'indiquer entre parenthèses pour faire prévaloir l'idée de relation.

15. Jean-Pierre Esquenazi, «Pouvoirs des séries télévisées », Éléments pour l'analyse des séries, op. cit., p. 90.

16. Raphaël Baroni et François Jost, « Repenser le récit avec les séries », dans Raphaël Baroni et François Jost (dir.), Télévision, $\mathrm{n}^{\circ}$ 7, 2016, p. 10.

17. Baroni, Jost, 2016.

18. Anne Besson, Constellations. Des mondes fictionnels dans l'imaginaire contemporain, Paris, CNRS, 2015, p. 12.

19. Besson, p. 21

20. Matthieu Letourneux, Fictions à la chaîne - Littératures sérielles et culture médiatique, Paris, Seuil, coll. « Poétique », 2017, p. 339. 
21. Ce que Philippe Marion nomme «transmédiagénie ». Cf. Philippe Marion, « Narratologie médiatique et médiagénie des récits ", Recherches en communication, $\mathrm{n}^{\circ}$ 7, 1997, p. 61-88.

22. Notamment en raison, selon Matthieu Letourneux, de l'intérêt pour les producteurs de décliner un univers préalablement sériel, ou encore de la stabilité du caractère encyclopédique de son architexte. Cf. Matthieu Letourneux, Fictions à la chaîne, p. 343.

23. Matthieu Letourneux, Fictions à la chaîne, p. 28.

24. Cf. François Jost, Introduction à l'analyse de la télévision, Paris, Ellipses, coll. « Infocom », 1999, p. 117.

25. Cf. Hélène Breda, op.cit.

26. Quinte-Curce, Histoires, Paris, Les Belles Lettres, coll. « CUF », (1948) 1965, Tome 2, p. 239.

27. Ibid., Tome 1, p. 4.

28. Pline l'Ancien, Histoire naturelle, livre XI, Paris, Les Belles Lettres, "CUF », 1947, p. 86.

29. Stace, Silves, Paris, Les Belles Lettres, « CUF », 1944, Tome 1, p. 116.

30. Quintilien, Institution oratoire, Paris, Librairie Garnier Frères, coll. « Classiques Garnier », 1933, Tome 2, p. 276-277.

31. Cicéron, De la divination, Paris, Librairie Garnier Frères, coll. "Classiques Garnier », 1937, p. 110-111.

32. Pour l'évolution historique des termes en français nous avons utilisé Alain Rey (dir.), Dictionnaire historique de la langue française, Paris, Dictionnaires Le Robert, (1993) 2010.

33. Aristote, Poétique, traduit par Barbara Gernez, Paris, Les Belles Lettres, coll. «Classiques en poche », (2001) 2008, p. 44-45.

34. La suite mathématique est un enchaînement de nombres suivant une loi connue, théorique et potentiellement infinie ; la série est une portion de cette suite, soit un enchaînement de nombres suivant la même loi mais fini, un outil qui permet d'examiner et de modéliser pratiquement l'évolution des suites.

35. Anonyme, «Série ou Suite: en Algèbre», dans Diderot, D’Alembert, L'Encyclopédie, Volume 15, 1765, p. 93b-96a : http://enccre.academie-sciences.fr/encyclopedie/article/

v15-174-0/, consulté le 19 février 2018.

36. Cf. Constant Guéroult, « Du roman-feuilleton », La Presse dramatique, $n^{\circ}$ 19, 28 décembre 1851, p. 1 : «On ne soupçonne pas les délices que procure aux départements la lecture quotidienne du roman-feuilleton; on ne sait pas avec quelle impatience est attendue cette suite dont le Parisien [...] remet la lecture à une époque de loisir qui n'arrive jamais ».

37. Anonyme, "Serial ", The Oxford English Dictionary, Oxford, Clarendon Press, (1933) 1961, Volume 9, p. 497.

38. Citons par exemple les Marches d'harmonie pratiquées dans la composition, ouvrage de L. Cherubini publié en 1847, dans lequel les harmonies simples sont décrites comme «établies sur des séries », en « formant une suite d'accords parfaits » (p. 103).

39. Sur ce point les exemples sont nombreux, de la lithographie inventée par Aloys Senefelder en 1796, à la photographie, en passant par la production d'appareils destinés à la commercialisation comme les lanternes magiques.

40. Cf. Anonyme, « Observations philatéliques ", Revue philatélique française, $n^{\circ} 85$, décembre 1897 , p. 149 : «Cette série si intéressante contient des variétés très curieuses », formulation montrant bien la cohérence d'un ensemble, au-delà de l'idée de production en série.

41. Le terme "série » est bien utilisé à l'époque pour désigner ses toiles : Cf. Camille Mauclair, L'Impressionnisme, son histoire, son esthétique, ses maîtres, Paris, Librairie de l'Art ancien et moderne, 1904, p. 73.

42. Cf. par exemple Thierry Lecointe, «Les premières années du spectacle cinématographique à Nîmes - 1895-1913 ", 1895, n 43, été 2004, p. 57.

43. Anonyme, "Éclair - Nick Carter, Roi des détectives », Ciné-Journal, n 5, 15 septembre 1908, p. 4 (nous soulignons). 
44. Ibid.

45. Anonyme, "Lone Thief Appears and Opposes "The Vampires" in Third Episode ", Motion Picture News, Volume 14, n² 22, 2 décembre 1916, p. 3490.

46. Anonyme, «"Give Them What They Don't Expect" - Feuillade », Motion Picture News, vol. 14, n 20, 18 novembre 1916, p. 3163.

47. Olivier Aïm, « La série télévisée comme machine à voir ", Entrelacs, Hors-série " Séries télé in/ out », 2008, mis en ligne le $1^{\text {er }}$ août 2012, consulté le 15 février 2018:: http:// entrelacs.revues.org/260.

48. Groupe $\mu$, Rhétorique de la poésie - Lecture linéaire, lecture tabulaire, Bruxelles, Éditions Complexe, 1977.

49. Cf. Georges Didi-Huberman, L'image survivante - Histoire de l'art et temps des fantômes selon Aby Warburg, Paris, Minuit, coll. " Paradoxes ", 2002 ; Philippe-Alain Michaud, Aby Warburg et l'image en mouvement, Paris, Macula, (1998) 2012 ; Erwin Panofsky, Essais d'iconologie - Thèmes humanistes dans l'art de la Renaissance, Paris, Gallimard, (1939) 1967.

50. AMC, 2008-2013.

51. HBO, 2002-2008.

52. HBO, 2011-2019.

53. Georges Perec, Tentative d'épuisement d'un lieu parisien, Paris, UGE, 1975 ; La vie mode d'emploi. Romans, Paris, Hachette, 1978.

54. Franco Moretti, Graphes, cartes et arbres - Modèles abstraits pour une autre histoire de la littérature, Paris, Les Prairies ordinaires, coll. « Penser/Croiser », 2008.

55. Publié en 1963, Marelle est un roman qui peut se lire selon deux types de trajectoires: de façon linéaire, page après page et du chapitre 1 au chapitre 56 ; ou bien de façon tabulaire en partant du chapitre 73 et suivant un ordre non consécutif indiqué au début du roman, à la façon des « livres dont vous êtes le héros », et privilégiant les excursus, les digressions, les glissements de la forme romanesque à d'autres formes littéraires.

56. Comme c'est régulièrement le cas lorsque TF1 par exemple diffuse des épisodes d'une série dans le désordre. Paresse du programmateur, ou génie du montage?

57. Dominique Avron, Le scintillant - Essai sur le phénomène télévisuel, Strasbourg, Presses universitaires de Strasbourg, 1994.

58. Thierry Lancien, « Un objet improbable », MédiaMorphoses, $n^{\circ}$ 6, "La télévision : média de la parole?», novembre 2002, p. 21.

59. L'un des exemples les plus célèbres est la série Community (NBC puis Yahoo Screens, 2009-2015), dans laquelle l'un des personnages principaux, Abed, est le seul à se savoir dans une série télévisée. Il est d'ailleurs significatif que ce personnage soit dès lors traité comme atteint d'une forme d'autisme prononcé, voire de psychose, comme si ce savoir réflexif était d'ordre nécessairement pathologique. On en revient toujours, au fond, à l'idée que faire tomber le mur entre le spectateur est le spectacle est un geste prohibé et dangereux...

\section{RÉSUMÉS}

Les approches narratologiques des séries télévisées tendent à occulter les richesses et les innovations formelles dont font preuve ces objets audiovisuels, dont la complexité dépasse le cadre strictement narratif. Le succès des approches narratologiques, dont la pertinence n'est 
nullement mise en cause, loin s'en faut, s'explique historiquement par les voies de son intégration dans le champ universitaire et les discours qui en déterminent la réception. Des méthodes développées dans d'autres champs disciplinaires, concernant d'autres arts ou objets culturels, peuvent cependant être sollicitées pour expliquer et rendre compte de la richesse et du succès des séries télévisées. Nous chercherons ici à poser les bases de cette réflexion, conçue comme un exercice d'ouverture du regard.

Narrative studies tend to detract television shows from their rich aesthetic value and inherent complexity, which is not limited to their narrative dimension. The success of narrative approaches, whose relevance is not in question here, can be explained historically by the way they were introduced in the academic field and by the discourses surrounding television shows and other forms of serials. Methods developed in other academic fields can however be called for to better explain and understand the aesthetic value and fame of television shows. In this article, we aim at laying the groundwork of this reflection and opening the way we look at and understand these objects.

\section{INDEX}

Keywords : narratology, seriality, arts, literature, cinema, painting

Mots-clés : narratologie, sérialité, arts, littérature, cinéma, peinture

\section{AUTEUR}

\section{SAUTE-REQUIN}

Saute-Requin est une association de chercheur-se's et étudiant·e's de divers horizons, toutes et tous passionnée's de formes sérielles, savantes ou populaires. Son objectif est de promouvoir la recherche et les activités culturelles autour des cultures sérielles. Cet article est le fruit du travail collaboratif de sept d'entre eux, armé'e-s des connaissances et méthodes de leurs champs disciplinaires respectifs : Sophie Barel, en sciences de l'information et de la communication ; Quentin Fischer et Jean Gueguen en littérature comparée ; Damien Keller, Yannick Kernec'h, Jean-Baptiste Massuet et Éric Thouvenel en études cinématographiques.

Saute-Requin (Jump-the-shark) is a collective composed of students and scholars from diverse backgrounds, all interested in serial cultural productions, whether erudite or popular. Its main purpose is to promote research and cultural activities about serial cultures. This article is the result of a collaborative work of seven of them, equipped with the tools and perspectives from their various disciplinary fields: Sophie Barel in communication studies, Quentin Fischer and Jean Gueguen in comparative literature; Damien Keller, Yannick Kernec'h, Jean-Baptiste Massuet and Éric Thouvenel in film studies 